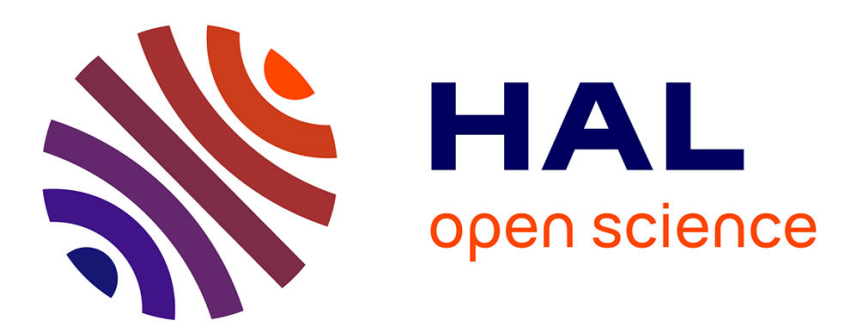

\title{
Consistency of Generalized Finite Difference Schemes for the Stochastic HJB Equation
}

\author{
J. Frederic Bonnans, Hasnaa Zidani
}

\section{To cite this version:}

J. Frederic Bonnans, Hasnaa Zidani. Consistency of Generalized Finite Difference Schemes for the Stochastic HJB Equation. [Research Report] RR-4162, INRIA. 2001. inria-00072460

\section{HAL Id: inria-00072460 \\ https://hal.inria.fr/inria-00072460}

Submitted on 24 May 2006

HAL is a multi-disciplinary open access archive for the deposit and dissemination of scientific research documents, whether they are published or not. The documents may come from teaching and research institutions in France or abroad, or from public or private research centers.
L'archive ouverte pluridisciplinaire HAL, est destinée au dépôt et à la diffusion de documents scientifiques de niveau recherche, publiés ou non, émanant des établissements d'enseignement et de recherche français ou étrangers, des laboratoires publics ou privés. 
INSTITUT NATIONAL DE RECHERCHE EN INFORMATIQUE ET EN AUTOMATIQUE

\title{
Consistency of generalized finite difference schemes for the stochastic HJB equation
}

\author{
J.F. Bonnans - H. Zidani
}

$\mathbf{N}^{\circ} 4162$

Avril 2001

THÈME 4 



\title{
Consistency of generalized finite difference schemes for the stochastic HJB equation
}

\author{
J.F. Bonnans* ${ }^{*}$ H. Zidani ${ }^{\dagger}$ \\ Thème 4 - Simulation et optimisation \\ de systèmes complexes \\ Projet SYDOCO \\ Rapport de recherche $\mathrm{n}^{\circ} 4162$ - Avril 2001 - 15 pages
}

\begin{abstract}
We analyse a class of numerical schemes for solving the HJB equation for stochastic control problems, that generalizes the usual finite difference method. The latter is known to be monotonous, and hence valid, only if the scaled covariance matrix is diagonal dominant. We generalize this result by, given the set of neighbouring points allowed to enter in the scheme, showing how to compute the class of covariance matrices that is consistent with this set of points. We perform this computation for several cases in dimension 2 to 4 .
\end{abstract}

Key-words: Stochastic control, finite differences, Hamilton Jacobi Bellman equation, consistency, numerical analysis of partial differential equations

* Inria-Rocquencourt, Domaine de Voluceau, BP 105, 78153 Le Chesnay, France. Email: Frederic.Bonnans@inria.fr

† Département de Mathématiques, Université d'Orléans, Laboratoire MAPMO, UFR Sciences, BP 6759, 45067 Orléans, France, and INRIA-Rocquencourt, France. Email: Housnaa.Zidani@inria.fr 


\section{Consistence des schémas de différences finies généralisées pour l'équation HJB stochastique}

Résumé : Nous analysons une classe de schémas numériques pour la résolution de l'équation HJB de la commande stochastique, qui généralise les méthodes usuelles de différences finies. Ces dernières ne sont monotones, et donc valides, que si la matrice de covariance mise à l'échelle est diagonale dominante. Nous généralisons ce résultat en fournissant, étant donné l'ensemble des points voisins pouvant intervenir dans le schéma, moyen de calculer la classe de matrices de covariances consistante cet ensemble de points. Nous avons effectué le calcul pour plusieurs cas, de dimension 2 à 4 .

Mots-clés : Commande stochastique, différences finies, équation de Hamilton Jacobi Bellman, consistence, analyse numérique d'équations aux dérivées partielles 


\section{Motivation}

This paper is devoted to the discussion of numerical algorithms for solving stochastic optimal control problems of the form (Fleming and Rishel [3], Lions and Bensoussan [6])

$$
\left\{\begin{array}{l}
\operatorname{Min} \mathbb{E} \int_{0}^{\infty} \ell(y(t), u(t)) e^{-\lambda t} d t ; \\
\left\{\begin{array}{l}
d y(t)=f(y(t), u(t)) d t+\sigma(y(t), u(t)) d w(t), \\
y(0)=x,
\end{array}\right. \\
u(t) \in U, t \in[0, \infty[.
\end{array}\right.
$$

In this problem $y(t) \in \mathbb{R}^{n}$ is the state variable, $u(t) \in \mathbb{R}^{m}$ is the control variable, $\lambda \geq 0$ is the discounting factor, $\ell: \mathbb{R}^{n} \times \mathbb{R}^{m} \rightarrow \mathbb{R}$ is a distributed cost, $f: \mathbb{R}^{n} \times \mathbb{R}^{m} \rightarrow \mathbb{R}^{n}$ is a deterministic dynamics, $\sigma(\cdot)$ is a mapping from $\mathbb{R}^{n} \times \mathbb{R}^{m}$ into the space of $n \times r$ matrices, and $w$ is a standard $r$ dimensional Brownian motion. We are assuming full observation of the state, and are looking for a control in the class of feedback controls.

Assuming the functions $f, \sigma$, and $\ell$, to be Lipschitz and bounded, the solution to the stochastic differential equation and associated cost are well defined. The covariance matrix (up to the factor $\frac{1}{2}$ ) is

$$
a(x, u):=\frac{1}{2} \sigma(x, u) \sigma(x, u)^{T}, \quad \forall(x, u) \in \mathbb{R}^{n} \times \mathbb{R}^{m} .
$$

It is known (see P.L. Lions [7], and also Fleming and Soner [4]) that the value function $V$ of problem $\left(P_{x}\right)$ is the unique bounded viscosity solution of the Hamilton-Jacobi-Bellman (HJB) equation

$$
\lambda v(x)=\mathcal{H}\left(x, v_{x}(x), v_{x x}(x)\right), \quad \text { for all } x \in \mathbb{R}^{n} ;
$$

where the Hamiltonian is defined as

$$
\mathcal{H}(x, p, Q):=\inf _{u \in U}\left\{\ell(x, u)+f(x, u) \cdot p+\sum_{i, j=1}^{n} a_{i j}(x, u) Q_{i j}\right\},
$$

where $x \in \mathbb{R}^{n}, p \in \mathbb{R}^{n}$, and $Q$ is a $n \times n$ symmetric matrix. A standard way for solving the second order, nonlinear partial differential equation (1.2) is to discretize the state space, and then to approximate the first and second order derivatives by finite differences. We develop these ideas in the next section.

\section{Generalized finite differences}

Let us present a generalization of the usual finite difference schemes, that in fact is nothing that another presentation of the class of Markov chain approximation discussed in

$\mathrm{RR} \mathrm{n}^{\circ} 4162$ 
Kushner and Dupuis [5]. We consider a regular grid of discretization of the state space $\mathbb{R}^{n}$, with discretization steps $h_{1}, \ldots, h_{n}$. With the coordinate $k=\left(k_{1}, \ldots, k_{n}\right)$ in $\mathbb{Z}^{n}$ is associated the point $x_{k} \in \mathbb{R}^{n}$ of the form

$$
x_{k}:=\left(k_{1} h_{1}, \ldots, k_{n} h_{n}\right) .
$$

Let $\varphi=\left\{\varphi_{k}\right\}$ be a real valued function over $\mathbb{Z}^{n}$. With $\xi \in \mathbb{Z}^{n}$, associate the shift operator $\delta_{\xi}$ defined by $\delta_{\xi} \varphi_{k}:=\varphi_{\xi+k}$. Consider the second order finite difference operator $\Delta_{\xi}=$ $\delta_{\xi}+\delta_{-\xi}-2 \delta_{0}$, in other words,

$$
\Delta_{\xi} \varphi_{k}:=\varphi_{k+\xi}+\varphi_{k-\xi}-2 \varphi_{k}=\varphi_{k+\xi}-\varphi_{k}-\left(\varphi_{k}-\varphi_{k-\xi}\right) .
$$

If $\Phi$ is a smooth function over $\mathbb{R}^{n}$, and $\varphi_{k}=\Phi\left(x_{k}\right)$ for all $k$, then by a standard Taylor expansion, we have that

$$
\Delta_{\xi} \varphi_{k}:=\sum_{i, j=1}^{n} h_{i} h_{j} \xi_{i} \xi_{j} \Phi_{x_{i} x_{j}}+o\left(\|h\|^{2}\right) .
$$

For instance, when $\xi$ is equal to $e_{i}$ (the $i$ th element of the natural basis of $\mathbb{R}^{n}$ ) and $e_{i} \pm e_{j}$, resp., we obtain

$$
\begin{cases}\Delta_{e_{i}} \varphi_{k} & =\left(h_{i}\right)^{2} \Phi_{x_{i} x_{i}}+o\left(\|h\|^{2}\right) \\ \Delta_{e_{i} \pm e_{j}} \varphi_{k} & =\left(h_{i}\right)^{2} \Phi_{x_{i} x_{i}}+\left(h_{j}\right)^{2} \Phi_{x_{j} x_{j}} \pm 2 h_{i} h_{j} \Phi_{x_{i} x_{j}}+o\left(\|h\|^{2}\right) .\end{cases}
$$

Denote by $v_{k}$ the approximation of the value function $V$ at $x_{k}$, and let $\mathcal{S}$ be a finite set of $\mathbb{Z}^{n}$. We consider explicit schemes based on the difference operators that we just discussed, namely

$$
\lambda v_{k}=\inf _{u \in U}\left\{\ell\left(x_{k}, u\right)+f\left(x_{k}, u\right) \cdot D_{k}^{u} v_{k}+\sum_{\xi \in \mathcal{S}} \alpha_{k, \xi}^{u} \Delta_{\xi} v_{k}\right\},
$$

for all $k \in \mathbb{Z}^{n}$. Here $D_{k}^{u} v_{k}$ is a notation for the upwind spatial finite difference,

$$
\left(D_{k}^{u} v_{k}\right)_{i}=\frac{v_{k+e_{i}}-v_{k}}{h_{i}} \text { if } f\left(x_{k}, u\right)_{i} \geq 0, \frac{v_{k}-v_{k-e_{i}}}{h_{i}} \text { if not. }
$$

Let $h_{0}>0$ denote a fictitious time step. Multiplying (2.7) by $h_{0}$ and adding $v_{k}$ on both sides, we get

$$
\begin{aligned}
v_{k} & :=\left(1+\lambda h_{0}\right)^{-1} \\
& \inf _{u \in U}\left\{v_{k}+h_{0} \ell\left(x_{k}, u\right)+h_{0} f\left(x_{k}, u\right) \cdot D_{k}^{u} v_{k}+h_{0} \sum_{\xi \in \mathcal{S}} \alpha_{k, \xi}^{u} \Delta_{\xi} v_{k}\right\} .
\end{aligned}
$$

The scheme is said to be monotonous if, for small enough $h_{0}$, the operator corresponding to the right hand side is a nondecreasing function of $v$. Since an infimum of nondecreasing 
functions is nondecreasing, a sufficient condition for monotonicity of the scheme is that, for each $u \in U$, the function in the r.h.s., i.e.,

$$
v \rightarrow v_{k}+h_{0} \ell\left(x_{k}, u\right)+h_{0} f\left(x_{k}, u\right) \cdot D_{k}^{u} v_{k}+h_{0} \sum_{\xi \in \mathcal{S}} \alpha_{k, \xi}^{u} \Delta_{\xi} v_{k}
$$

is nondecreasing. We call this a uniform monotonicity condition. Since (2.11) is an affine function of $v$, it is easily checked that the uniform monotonicity condition holds iff

$$
\begin{array}{r}
\alpha_{k, \xi}^{u} \geq 0, \quad \forall(\xi, k, u) \in \mathcal{S} \times \mathbb{Z}^{n} \times U . \\
\sum_{i=1}^{n} \frac{\left|f_{i}\left(x_{k}, u\right)\right|}{h_{i}}+2 \sum_{\xi \in \mathcal{S}} \alpha_{k, \xi}^{u} \leq h_{0}^{-1}, \quad \forall(k, u) \in \mathbb{Z}^{n} \times U .
\end{array}
$$

The second condition is always satisfied when $h_{0}$ is small enough, once a (uniform in space) estimate of $\sum_{\xi \in \mathcal{S}} \alpha_{k, \xi}^{u}$ is known.

The scheme is said to be consistent if, for all smooth function $\Phi$ over $\mathbb{R}^{n}$, and $k \in \mathbb{Z}^{n}$, we have

$$
\sum_{\xi \in \mathcal{S}} \alpha_{k, \xi}^{u} \Delta_{\xi} \Phi\left(x_{k}\right)=\sum_{i, j=1}^{n} a_{i j}\left(x_{k}, u\right) \Phi_{x_{i} x_{j}}\left(x_{k}\right)+o(1) .
$$

It is known that those two assumptions of monotonicity and consistency allow to prove the convergence of the solution to the numerical scheme towards the solution of the original problem, see Barles and Souganidis [2]. In the sequel we will assume (2.9)-(2.13) to be satisfied, so that the scheme is monotone, and we therefore concentrate on the consistency condition. Let $a^{h}$ denote the scaled covariance matrix $\left\{a_{i j} / h_{i} h_{j}\right\}$. In view of (2.6), consistency holds iff we have

$$
\sum_{\xi \in \mathcal{S}} \alpha_{k, \xi}^{u} \xi \xi^{T}=a^{h}\left(x_{k}, u\right)+o(1), \quad \text { for all } k \in \mathbb{Z}^{n} .
$$

In the sequel, we discuss the strong consistency property

$$
\sum_{\xi \in \mathcal{S}} \alpha_{k, \xi}^{u} \xi \xi^{T}=a^{h}\left(x_{k}, u\right), \quad \text { for all } k \in \mathbb{Z}^{n}
$$

Since $\alpha$ is nonnegative, strong consistency means that the symmetric matrix $a^{h}\left(x_{k}, u\right)$ belongs, for all $k$ and $u$, to the cone generated by the set $\left\{\xi \xi^{T} ; \xi \in \mathcal{S}\right\}$ that we denote

$$
\mathcal{C}(\mathcal{S}):=\left\{\sum_{\xi \in \mathcal{S}} \alpha_{\xi} \xi \xi^{T} ; \alpha \in \mathbb{R}_{+}^{|\mathcal{S}|}\right\} .
$$

Note that strong consistency implies a bound on the coefficients $\alpha_{k, \xi}^{u}$, which in turn allows to obtain an estimate of the fictitious time step. 
Lemma 2.1 Assume that the strong consistency condition holds. Then

$$
\sum_{\xi \in \mathcal{S}} \alpha_{k, \xi}^{u} \leq \operatorname{trace} a^{h}\left(x_{k}, u\right)
$$

and hence, condition (2.13) for the fictitious time step is satisfied whenever

$$
\sum_{i=1}^{n} \frac{\left\|f_{i}\right\|_{\infty}}{h_{i}}+2 \operatorname{trace}\left\|a^{h}\right\|_{\infty} \leq h_{0}^{-1}
$$

It follows from this lemma that, when $h \rightarrow 0$, we have that $h_{0}$ is of order $O\left(\min _{i} h_{i}^{2}\right)$, as expected.

\section{Classical finite differences approximations}

Let us show that the above generalized finite difference algorithm is indeed a generalization of the classical finite differences approximations, that we recall now. Let $\Phi$ be a smooth function over $\mathbb{R}^{n}$, and let $\varphi_{k}:=\Phi\left(x_{k}\right)$ for all $k$. Given any $\xi \in \mathbb{Z}^{n}$, we can approximate the second order derivatives of $\Phi$ by the following second order finites differences:

$$
\frac{\delta_{\xi+e_{i}+e_{j}}-\delta_{\xi+e_{i}}-\delta_{\xi+e_{j}}+\delta_{\xi}}{h_{i} h_{j}} \varphi_{k}=\Phi_{x_{i} x_{j}}\left(x_{k}\right)+o(1) .
$$

Denote the corresponding operators as follows:

$$
d_{i j}^{\xi}:=\frac{\delta_{\xi+e_{i}+e_{j}}-\delta_{\xi+e_{i}}-\delta_{\xi+e_{j}}+\delta_{\xi}}{h_{i} h_{j}} .
$$

Viewing $i$ (resp. $j$ ) as the first (second) coordinate, when $\xi=0$, we call this operator $d_{i j}^{\xi}$ the right upper approximation of $\Phi_{x_{i} x_{j}}$. We can similarly define left upper, right lower, and left lower approximations of $\Phi_{x_{i} x_{j}}$, by taking $\xi$ equal to $-e_{i},-e_{j}$, and $-e_{i}-e_{j}$, resp. By combining these amounts, we can define centered approximations; the corresponding operators are, along the main and second diagonals:

$$
D_{i j}^{+}:=\frac{1}{2}\left(d_{i j}^{0}+d_{i j}^{-e_{i}-e_{j}}\right), \quad D_{i j}^{-}:=\frac{1}{2}\left(d_{i j}^{-e_{i}}+d_{i j}^{-e_{j}}\right) .
$$

In other words,

$$
\begin{aligned}
& D_{i j}^{+}=\frac{1}{2 h_{i} h_{j}}\left(\delta_{e_{i}+e_{j}}+\delta_{-e_{i}-e_{j}}+2 \delta_{0}-\delta_{e_{i}}-\delta_{-e_{i}}-\delta_{e_{j}}-\delta_{-e_{j}}\right), \\
& D_{i j}^{-}=\frac{1}{2 h_{i} h_{j}}\left(\delta_{e_{i}}+\delta_{-e_{i}}+\delta_{e_{j}}+\delta_{-e_{j}}-\delta_{e_{i}-e_{j}}-\delta_{e_{j}-e_{i}}-2 \delta_{0}\right) .
\end{aligned}
$$


In addition, for the approximation of diagonal second order derivatives we take the standard centered formula

$$
D_{i i}:=\frac{\delta_{e_{i}}+\delta_{-e_{i}}-2 \delta_{0}}{h_{i} h_{i}}
$$

The classical finite differences approximation of (1.2) is

$$
\begin{aligned}
\lambda v_{k}= & \inf _{u \in U}\left(\ell\left(x_{k}, u\right)+f\left(x_{k}, u\right) \cdot D_{k}^{u} v_{k}+\sum_{i, j=1}^{n} a_{i j}\left(x_{k}, u\right) D_{i j} v_{k}\right) \\
& \quad \text { for all } k \in \mathbb{Z}^{n}, q \in \mathbb{N}, \\
y_{k}^{0}= & 0, \quad \text { for all } k \in \mathbb{Z}^{n},
\end{aligned}
$$

where if $i \neq j, D_{i j}$ is equal either to $D_{i j}^{+}$or $D_{i j}^{-}$, and $D_{k}^{u}$ is the the upwind spatial finite difference defined in (2.9). This is another scheme where the second order term is approximated by using a combination or neighboring points. The corresponding consistency condition

$$
\sum_{i, j=1}^{n} a_{i j}\left(x_{k}, u\right) D_{i j} \varphi\left(x_{k}, u\right)=\sum_{i, j=1}^{n} a_{i j}\left(x_{k}, u\right) \Phi_{x_{i} x_{j}}\left(x_{k}, u\right)+o(1)
$$

is always satisfied. However, the monotonicity condition holds under restrictive assumptions that we explicit now (this is a reformulation of known results; see e.g. Lions and Mercier $[8])$.

Lemma 3.1 The classical finite differences approximation scheme is uniformly monotone iff the following three conditions hold:

(i) If $i \neq j$ is such that $a_{i j}\left(x_{k}, u\right) \neq 0$, then $D_{i j}=D_{i j}^{+}$if $a_{i j}\left(x_{k}, u\right)>0$, and $D_{i j}=D_{i j}^{-}$if $a_{i j}\left(x_{k}, u\right)<0$,

(ii) $a^{h}\left(x_{k}, u\right)$ is diagonal dominant, or equivalently,

$$
\frac{a_{i i}\left(x_{k}, u\right)}{h_{i}} \geq \sum_{j \neq i} \frac{\left|a_{i j}\left(x_{k}, u\right)\right|}{h_{j}} \quad \text { for all } i=1, \ldots, n
$$

(iii) The time step $h_{0}$ satisfies the following condition

$$
\sum_{i=1}^{n} \frac{\left|f\left(x_{k}, u\right)_{i}\right|}{h_{i}}+\sum_{i=1}^{n}\left(2 \frac{a_{i i}\left(x_{k}, u\right)}{h_{i}^{2}}-\sum_{j \neq i} \frac{\left|a_{i j}\left(x_{k}, u\right)\right|}{h_{i} h_{j}}\right) \leq h_{0}^{-1} .
$$

Proof. Let $i \neq j$. Consider first the weight of terms of the form $v_{k \pm e_{i} \pm e_{j}}$. If $a_{i j}\left(x_{k}, u\right)=0$, this weight is 0 . Otherwise, by (3.23), the weight is of the sign of $a_{i j}\left(x_{k}, u\right)$ if $D_{i j}=D_{i j}^{+}$, and 
of the opposite sign if $D_{i j}=D_{i j}^{-}$. Therefore (i) is a necessary condition for monotonicity of the scheme. If (i) is satisfied, then the weight of terms of the form $v_{k \pm e_{i}}$ is

$$
\frac{a_{i i}\left(x_{k}, u\right)}{h_{i}^{2}}-\sum_{j \neq i} \frac{\left|a_{i j}\left(x_{k}, u\right)\right|}{h_{i} h_{j}} .
$$

This weight is nonnegative iff (3.27) holds. Finally the weight of $v_{0}$ if nonnegative iff (3.28) holds.

We now make explicit the link between the two approaches by expressing the classical finite differences approximation scheme as a Markov chain approximation scheme. If the conditions of the above lemma are satisfied, then we can write the approximation of second order terms as

$$
\begin{aligned}
\sum_{i, j=1}^{n} a_{i j}\left(x_{k}, u\right) D_{i j}= & \frac{1}{2} \sum_{\substack{i \neq j \\
a_{i j}>0}} \frac{a_{i j}}{h_{i} h_{j}} \Delta_{e_{i}+e_{j}}-\frac{1}{2} \sum_{\substack{i \neq j \\
a_{i j}<0}} \frac{a_{i j}}{h_{i} h_{j}} \Delta_{e_{i}-e_{j}}+ \\
& \sum_{i}\left(\frac{a_{i i}}{\left(h_{i}\right)^{2}}-\sum_{j \neq i} \frac{\left|a_{i j}\right|}{h_{i} h_{j}}\right) \Delta_{e_{i}}
\end{aligned}
$$

The weights of the transitions are nonnegative iff condition (ii) of lemma 3.1 is satisfied. It follows that the classical finite difference scheme is equivalent to a Markov chain approximation with $\mathcal{S}$ here equal to $\mathcal{S}_{2}$.

\section{Characterization of finitely generated cones}

Let us come back to the analysis of the generalized finite difference method. In the sequel we will concentrate on characterizations of the strong consistence condition, with special attention to the case when $\mathcal{S}$ is the set $\mathcal{S}^{q}$ of neighboring points of order $q$, defined by

$$
\mathcal{S}^{q}:=\left\{\xi \in \mathbb{Z}^{n} ;\left|\xi_{i}\right| \leq q, i=1, \ldots, n\right\} .
$$

Characterizing a finitely generated cone happens to be a classical problem of convex analysis and polyhedral combinatorics, and can be solved using the notion of polar cone. Let us recall these classical results; an excellent reference on this subject is Pulleyblank [9].

Let $\mathcal{C}$ be a nonempty closed convex cone in $\mathbb{R}^{p}$. The associated (positively) polar cone is

$$
\mathcal{C}^{*}=\left\{x^{*} \in X^{*} ;\left\langle x^{*}, x\right\rangle \geq 0, \forall x \in \mathcal{C}\right\} .
$$

It is known that $\left(\mathcal{C}^{*}\right)^{*}=\mathcal{C}$. Let $\mathcal{C}$ be finitely generated, say by $g_{1}, \ldots, g_{q}$. Then $\mathcal{C}^{*}=$ $\bigcap_{i}\left\{x^{*} \in X^{*} ;\left\langle x^{*}, g_{i}\right\rangle \geq 0\right\}$. It happens that the set $\mathcal{C}^{*}$ is also finitely generated, say by 
$g_{1}^{*}, \ldots, g_{r}^{*}$; this dual generator can be computed by a certain recursion. Since $\mathcal{C}=\left(\mathcal{C}^{*}\right)^{*}$, it follows that

$$
\mathcal{C}=\left\{x ;\left\langle g_{i}^{*}, x\right\rangle \geq 0, \quad i=1, \ldots, r\right\} .
$$

This means that the cone $\mathcal{C}$ is characterized by a finite number of linear inequalities, whose coefficients can be computed.

Let us specialize this result to the case of the cone $\mathcal{C}(\mathcal{S})$. Let $\mathcal{M}$ be the set of symmetric matrices, and $\mathcal{M}_{+}$be the set of symmetric definite positive matrices. Using the Frobenius scalar product $A \cdot B=\sum_{i, j} A_{i j} B_{i j}$, for which $B \cdot \xi \xi^{T}=\xi^{T} B \xi$, for all square $n \times n$ symmetric matrix $B$ and $n$ dimensional vector $\xi$, we have that the polar cone is

$$
\mathcal{C}\left(\mathcal{S}_{p}\right)^{*}=\left\{B \in \mathcal{M} ; \xi^{T} B \xi \geq 0, \forall \xi \in \mathcal{S}\right\} .
$$

Consider the example when $\mathcal{S}=\mathcal{S}^{q}$ defined in (4.31). Using the following facts: $\mathcal{C}\left(\mathcal{S}^{q}\right)$ is strictly increasing with $q$, is a subset of the cone $\mathcal{M}_{+}$, and $\left(\mathcal{M}_{+}\right)^{*}=\mathcal{M}_{+}$, we have the infinite chain of strict inclusions

$$
\mathcal{C}\left(\mathcal{S}^{1}\right) \subset \mathcal{C}\left(\mathcal{S}^{2}\right) \cdots \subset \mathcal{M}_{+} \subset \cdots \mathcal{C}\left(\mathcal{S}^{2}\right)^{*} \subset \mathcal{C}\left(\mathcal{S}_{1}\right)^{*} .
$$

It can be noticed that, since the cone $\mathcal{C}\left(\mathcal{S}^{q}\right)$ contains every nonnegative diagonal matrices, each element of its dual has a nonnegative diagonal.

An important observation is that $\mathcal{S}^{q}$, and therefore also $\mathcal{C}\left(\mathcal{S}^{q}\right)$, are invariant through the linear transformations in $\mathbb{R}^{n}$ that correspond to a permutation of coordinates, and also to the change of sign of coordinates. The permutation of coordinates $i$ and $j$ of $\xi \in \mathbb{R}^{n}$ result in the permutation of elements of $\xi \xi^{T}$ of coordinates $(i, k)$ and $(j, k)$, and $(k, i)$ and $(k, j)$, for all $k$, while changing the sign of $\xi_{i}$ results in changing the sign of elements of $\xi \xi^{T}$ of coordinates $(i, j)$, for $j \neq i$. Since these transformations are self adjoint, for each $B \in \mathcal{C}\left(\mathcal{S}^{q}\right)^{*}$, the matrices obtained by the same (adjoint) transformations (so that the scalar product with $B$ remains invariant) also belong to $\mathcal{C}\left(\mathcal{S}^{q}\right)^{*}$. In particular, a generator of $\mathcal{C}\left(\mathcal{S}^{q}\right)^{*}$ can be partitioned into classes of equivalence corresponding to the above mentioned transformations. This allows to give a compact description of the set of generators.

\section{$5 \quad$ Specific examples}

We have performed the computation of generators of dual cones using the Qhull algorithm by Barbet et al. [1]. The latter computes, given a finite set in $\mathbb{R}^{m}$, a minimal set of linear inequalities characterizing its convex hull. This computation is made using the floating point arithmetic of the $\mathrm{C}$ language. However, the risk of numerical errors due to the floating point arithmetic is limited, since we were able to compute a scaling of the data for which all coefficients are small integers, up to an absolute precision of $10^{-10}$.

The link between the convex hull of a finite set and the generator of a dual cone is as follows. Consider a generator $g_{1}, \ldots, g_{n}$, and set $g_{0}:=0$. Then compute a minimal characterization of the convex hull of $g_{0}, \ldots, g_{n}$, of the form $\left\langle g_{i}^{*}, \cdot\right\rangle \geq b_{i}, i=1, \ldots, r$. A 
minimal generator of the dual cone is given by the homogeneous inequalities, i.e., the dual cone is

$$
\left\{g \in \mathbb{R}^{m} ;\left\langle g_{i}^{*}, g\right\rangle \geq b_{i}, \quad i \in I\right\}
$$

with $I:=\left\{1 \leq i \leq r ; \quad b_{i}=0\right\}$.

Our actual computations deal with spaces of symmetric matrices of size $n$. Each of them can be represented by its upper triangular part, and thus is viewed as an element of $\mathbb{R}^{m}$, $m=\frac{1}{2} n(n+1)$; in particular, $m=3,6$, and 10 , for $n=2,3$, and 4 , respectively.

Once a generator of the dual cone has been obtained, it remains to identify the classes of equivalence in order to obtain compact expression. This was done by sorting the elements following the (ordered) weights of diagonal elements (the latter being, as we already know, nonnegative). It appears that this suffices for identifying the equivalence classes, as can be checked by generating them using the formulas given below and comparing both sets.

Dimension 2. In the case $n=2$, we computed characterizations of the sets $\mathcal{C}\left(\mathcal{S}^{q}\right), q=1$ to 10 . We display detailed results for $q=1$ to 5 . The set $\mathcal{C}\left(\mathcal{S}^{1}\right)$ is characterized by 4 constraints and 1 equivalence class:

$$
a_{i i} \geq\left|a_{i j}\right|, \quad 1 \leq i \neq j \leq 2 .
$$

The set $\mathcal{C}\left(\mathcal{S}^{2}\right)$ is characterized by 8 constraints and 2 equivalence classes:

$$
\left\{\begin{array}{l}
2 a_{i i} \geq\left|a_{i j}\right| \\
2 a_{i i}+a_{j j} \geq 3\left|a_{i j}\right|
\end{array}\right.
$$

for $1 \leq i \neq j \leq 2$. The $\operatorname{set} \mathcal{C}\left(\mathcal{S}^{3}\right)$ is characterized by 16 constraints and 4 equivalence classes:

$$
\left\{\begin{array}{l}
3 a_{i i} \geq\left|a_{i j}\right| \\
3 a_{i i}+2 a_{j j} \geq 5\left|a_{i j}\right| . \\
6 a_{i i}+a_{j j} \geq 5\left|a_{i j}\right| . \\
6 a_{i i}+2 a_{j j} \geq 7\left|a_{i j}\right| .
\end{array}\right.
$$

for $1 \leq i \neq j \leq 2$. The $\operatorname{set} \mathcal{C}\left(\mathcal{S}^{4}\right)$ is characterized by 24 constraints and 6 equivalence classes:

$$
\left\{\begin{array}{l}
4 a_{i i} \geq\left|a_{i j}\right| \\
4 a_{i i}+3 a_{j j} \geq 7\left|a_{i j}\right| \\
6 a_{i i}+a_{j j} \geq 5\left|a_{i j}\right| \\
6 a_{i i}+2 a_{j j} \geq 7\left|a_{i j}\right| \\
12 a_{i i}+a_{j j} \geq 7\left|a_{i j}\right| \\
12 a_{i i}+6 a_{j j} \geq 17\left|a_{i j}\right|
\end{array}\right.
$$

for $1 \leq i \neq j \leq 2$. The set $\mathcal{C}\left(\mathcal{S}^{5}\right)$ is characterized by 40 constraints and 10 equivalence classes: 


$$
\left\{\begin{array}{l}
5 a_{i i} \geq\left|a_{i j}\right| \\
5 a_{i i}+4 a_{j j} \geq 9\left|a_{i j}\right| \\
10 a_{i i}+2 a_{j j} \geq 9\left|a_{i j}\right| \\
10 a_{i i}+3 a_{j j} \geq 11\left|a_{i j}\right| \\
12 a_{i i}+a_{j j} \geq 7\left|a_{i j}\right| \\
12 a_{i i}+6 a_{j j} \geq 17\left|a_{i j}\right| \\
15 a_{i i}+2 a_{j j} \geq 11\left|a_{i j}\right| \\
15 a_{i i}+6 a_{j j} \geq 19\left|a_{i j}\right| \\
20 a_{i i}+a_{j j} \geq 9\left|a_{i j}\right| \\
20 a_{i i}+12 a_{j j} \geq 31\left|a_{i j}\right|
\end{array}\right.
$$

The set $\mathcal{C}\left(\mathcal{S}^{6}\right)$ is characterized by 48 constraints and 12 equivalence classes:

$$
\left\{\begin{array}{l}
6_{i i} \geq\left|a_{i j}\right| \\
6 a_{i i}+5 a_{j j} \geq 11\left|a_{i j}\right| \\
10 a_{i i}+2 a_{j j} \geq 9\left|a_{i j}\right| \\
10 a_{i i}+3 a_{j j} \geq 11\left|a_{i j}\right| \\
12 a_{i i}+a_{j j} \geq 7\left|a_{i j}\right| \\
12 a_{i i}+6 a_{j j} \geq 17\left|a_{i j}\right| \\
15 a_{i i}+2 a_{j j} \geq 11\left|a_{i j}\right| \\
15 a_{i i}+6 a_{j j} \geq 19\left|a_{i j}\right| \\
20 a_{i i}+a_{j j} \geq 9\left|a_{i j}\right| \\
20 a_{i i}+12 a_{j j} \geq 31\left|a_{i j}\right| \\
30 a_{i i}+a_{j j} \geq 11\left|a_{i j}\right| \\
30 a_{i i}+20 a_{j j} \geq 49\left|a_{i j}\right|
\end{array}\right.
$$


The set $\mathcal{C}\left(\mathcal{S}^{7}\right)$ is characterized by 72 constraints and 18 equivalence classes:

$$
\left\{\begin{array}{l}
7 a_{i i} \geq\left|a_{i j}\right| \\
7 a_{i i}+6 a_{j j} \geq 13\left|a_{i j}\right| \\
14 a_{i i}+3 a_{j j} \geq 13\left|a_{i j}\right| \\
14 a_{i i}+4 a_{j j} \geq 15\left|a_{i j}\right| \\
15 a_{i i}+2 a_{j j} \geq 11\left|a_{i j}\right| \\
15 a_{i i}+6 a_{j j} \geq 19\left|a_{i j}\right| \\
20 a_{i i}+a_{j j} \geq 9\left|a_{i j}\right| \\
20 a_{i i}+12 a_{j j} \geq 31\left|a_{i j}\right| \\
21 a_{i i}+2 a_{j j} \geq 13\left|a_{i j}\right| \\
21 a_{i i}+10 a_{j j} \geq 29\left|a_{i j}\right| \\
28 a_{i i}+2 a_{j j} \geq 15\left|a_{i j}\right| \\
28 a_{i i}+15 a_{j j} \geq 41\left|a_{i j}\right| \\
30 a_{i i}+a_{j j} \geq 11\left|a_{i j}\right| \\
30 a_{i i}+20 a_{j j} \geq 49\left|a_{i j}\right| \\
35 a_{i i}+6 a_{j j} \geq 29\left|a_{i j}\right| \\
35 a_{i i}+12 a_{j j} \geq 41\left|a_{i j}\right| \\
42 a_{i i}+a_{j j} \geq 13\left|a_{i j}\right| \\
42 a_{i i}+30 a_{j j} \geq 71\left|a_{i j}\right|
\end{array}\right.
$$

Dimension 3. When $n=3$, we computed characterizations of the sets $\mathcal{C}\left(\mathcal{S}^{q}\right), q=1$ to 2 . The set $\mathcal{C}\left(\mathcal{S}^{1}\right)$ is characterized by

$$
\left\{\begin{array}{l}
a_{i i} \geq\left|a_{i j}\right| \\
a_{i i}+a_{j j} \geq(-1)^{p} a_{i k}+(-1)^{q} a_{j k}+2(-1)^{p+q+1} a_{i j}
\end{array}\right.
$$


for $i \neq j \neq k$ and $p, q \in\{1,2\}$. The set $\mathcal{C}\left(\mathcal{S}^{2}\right)$ is characterized by

$$
\left\{\begin{array}{l}
2 a_{i i} \geq\left|a_{i j}\right| \\
2 a_{i i}+a_{j j} \geq 3\left|a_{i j}\right| \\
2 a_{i i}+2 a_{j j} \geq 4(-1)^{p} a_{i j}+(-1)^{q} a_{j k}-(-1)^{p+q} a_{i k} \\
2 a_{i i}+2 a_{j j}+a_{k k} \geq 4(-1)^{p} a_{i j}+3(-1)^{q} a_{j k}-3(-1)^{p+q} a_{i k} \\
3 a_{i i}+2 a_{j j}+2 a_{k k} \geq 5(-1)^{p} a_{i j}+4(-1)^{q} a_{j k}-5(-1)^{p+q} a_{i k} \\
6 a_{i i}+a_{j j}+a_{k k} \geq 5(-1)^{p} a_{i j}+2(-1)^{q} a_{j k}-5(-1)^{p+q} a_{i k} \\
6 a_{i i}+2 a_{j j}+a_{k k} \geq 7(-1)^{p} a_{i j}+3(-1)^{q} a_{j k}-5(-1)^{p+q} a_{i k} \\
6 a_{i i}+2 a_{j j}+2 a_{k k} \geq 7(-1)^{p} a_{i j}+4(-1)^{q} a_{j k}-7(-1)^{p+q} a_{i k} \\
8 a_{i i}+2 a_{j j} \geq 8(-1)^{p} a_{i j}+(-1)^{q} a_{j k}-2(-1)^{p+q} a_{i k} \\
8 a_{i i}+2 a_{j j}+a_{k k} \geq 8(-1)^{p} a_{i j}+3(-1)^{q} a_{j k}-6(-1)^{p+q} a_{i k} \\
8 a_{i i}+3 a_{j j}+2 a_{k k} \geq 10(-1)^{p} a_{i j}+5(-1)^{q} a_{j k}-8(-1)^{p+q} a_{i k} \\
8 a_{i i}+6 a_{j j}+2 a_{k k} \geq 14(-1)^{p} a_{i j}+7(-1)^{q} a_{j k}-8(-1)^{p+q} a_{i k} \\
12 a_{i i}+2 a_{j j}+a_{k k} \geq 10(-1)^{p} a_{i j}+3(-1)^{q} a_{j k}-7(-1)^{p+q} a_{i k} \\
12 a_{i i}+4 a_{j j}+a_{k k} \geq 14(-1)^{p} a_{i j}+4(-1)^{q} a_{j k}-7(-1)^{p+q} a_{i k} \\
12 a_{i i}+6 a_{j j}+2 a_{k k} \geq 17(-1)^{p} a_{i j}+7(-1)^{q} a_{j k}-10(-1)^{p+q} a_{i k} \\
12 a_{i i}+6 a_{j j}+4 a_{k k} \geq 17(-1)^{p} a_{i j}+10(-1)^{q} a_{j k}-14(-1)^{p+q} a_{i k} \\
18 a_{i i}+2 a_{j j}+a_{k k} \geq 12(-1)^{p} a_{i j}+3(-1)^{q} a_{j k}-9(-1)^{p+q} a_{i k} \\
18 a_{i i}+8 a_{j j}+a_{k k} \geq 24(-1)^{p} a_{i j}+6(-1)^{q} a_{j k}-9(-1)^{p+q} a_{i k} \\
18 a_{i i}+10 a_{j j}+2 a_{k k} \geq 27(-1)^{p} a_{i j}+9(-1)^{q} a_{j k}-12(-1)^{p+q} a_{i k}
\end{array}\right.
$$

Dimension 4. When $n=4$, the set $\mathcal{C}\left(\mathcal{S}^{1}\right)$ is characterized by

$$
\begin{cases}a_{i i} & \geq\left|a_{i j}\right| \\ a_{i i}+a_{j j} & \geq(-1)^{p} a_{i k}+(-1)^{q} a_{j k}-2(-1)^{p+q} a_{i j} \\ a_{i i}+a_{j j}+a_{k k} & \geq(-1)^{p} a_{i l}+(-1)^{q} a_{j l}+(-1)^{r} a_{k l} \\ & -2(-1)^{p+q} a_{i j}-2(-1)^{p+r} a_{i k}-2(-1)^{q+r} a_{j k} \\ 2 a_{i i}+a_{j j}+a_{k k}+a_{l l} & \geq 3(-1)^{p} a_{i j}+3(-1)^{q} a_{i k}+3(-1)^{r} a_{i l} \\ & -2(-1)^{p+q} a_{j k}-2(-1)^{p+r} a_{j l}-2(-1)^{q+r} a_{k l} \\ & \geq 2(-1)^{p} a_{i l}+(-1)^{q} a_{j l}+(-1)^{r} a_{k l} \\ 4 a_{i i}+a_{j j}+a_{k k} & -4(-1)^{p+q} a_{i j}-4(-1)^{p+r} a_{i k}-2(-1)^{q+r} a_{j k} \\ & \\ 4 a_{i i}+2 a_{j j}+a_{k k}+a_{l l} & \geq 6(-1)^{p} a_{i j}+4(-1)^{q} a_{i k}+4(-1)^{r} a_{i l} \\ & -3(-1)^{p+q} a_{j k}-3(-1)^{p+r} a_{j l}-2(-1)^{q+r} a_{k l}\end{cases}
$$

for $i \neq j \neq k \neq l$ and $p, q, r \in\{1,2\}$.

Summary of results. The following table summerizes the various steps of our calculation, and highlights the importance of reduction of constraints using the classes of equivalence. 


\begin{tabular}{|c|c|c|c|c|c|}
\hline$n$ & $q$ & $\begin{array}{c}\text { size of generator } \\
\text { of primal cone }\end{array}$ & $\begin{array}{c}\text { \# of constraints } \\
\text { defining } \mathcal{S}^{*}\end{array}$ & $\begin{array}{c}\text { \# of constraints } \\
\text { defining } \mathcal{C}\end{array}$ & $\begin{array}{c}\text { \# of classes } \\
\text { of equivalence }\end{array}$ \\
\hline \hline 2 & 1 & 4 & 6 & 4 & 1 \\
\hline 2 & 2 & 8 & 13 & 8 & 2 \\
\hline 2 & 3 & 16 & 27 & 16 & 4 \\
\hline 2 & 4 & 24 & 39 & 24 & 6 \\
\hline 2 & 5 & 40 & 67 & 40 & 10 \\
\hline 2 & 6 & 48 & 87 & 48 & 12 \\
\hline 2 & 7 & 72 & 123 & 72 & 18 \\
\hline 2 & 8 & 88 & 159 & 88 & 22 \\
\hline 2 & 9 & 112 & 203 & 112 & 28 \\
\hline 2 & 10 & 128 & 239 & 128 & 32 \\
\hline \hline 3 & 1 & 13 & 31 & 24 & 2 \\
\hline 3 & 2 & 49 & 563 & 372 & 19 \\
\hline \hline 4 & 1 & 40 & 476 & 328 & 6 \\
\hline
\end{tabular}

\section{Discussion of results}

In this paper we have clarified some aspects of the "Markov chain approximation" method discussed in Kushner and Dupuis [5]. We have presented a general method for checking the consistency condition. It seems that we have performed the computations for essentially all cases for which the numerical resolution of the stochastic HJB equation is of reasonable complexity.

On the other hand, our results are only a preliminary step towards an efficient numerical algorithm. There are two main difficulties. The first is to design fast algorithms for computing the coefficients $\alpha_{k, \xi}^{u}$. The latter are, by the definition, solution of a linear programming problem, but using a linear programming solver for each control, at each point of the grid would be inefficient. The second difficulty is to deal with the case when consistency does not hold, e.g. by approximating the matrix $a\left(x_{k}, u\right)$ by a consistent matrix. We are now pursuing some research in these directions.

\section{References}

[1] C.B. Barber, D.P. Dobkin, and H. Huhdanpaa. The quickhull algorithm for convex hulls. ACM Transactions for Mathematical Software, 22:469-483, 1996.

[2] G. Barles and P. E. Souganidis. Convergence of approximation schemes for fully nonlinear second order equations. Asymptotic Analysis, 4:271-283, 1991.

[3] W. H. Fleming and R. Rishel. Deterministic and stochastic optimal control, volume 1 of Applications of mathematics. Springer, New York, 1975. 
[4] W. H. Fleming and H.M. Soner. Controlled Markov processes and viscosity solutions. Springer, New York, 1992.

[5] H. J. Kushner and P. G. Dupuis. Numerical methods for stochastic control problems in continuous time, volume 24 of Applications of mathematics. Springer, New York, 1992.

[6] J. L. Lions and A. Bensoussan. Application des inéquations variationnelles en contrôle stochastique, volume 6 of Méthodes mathématiques de l'informatique. Dunod, Paris, 1978.

[7] P.L. Lions. Optimal control of diffusion processes and Hamilton-Jacobi-Bellman equations. Part 2: viscosity solutions and uniqueness. Communications in pure and applied mathematics, 8:1220-1276, 1983.

[8] P.L. Lions and B. Mercier. Approximation numérique des équations de Hamilton-JacobiBellman. RAIRO Analyse numérique, 14:369-393, 1980.

[9] W.R. Pulleyblank. Polyhedral combinatorics. In G.L. Nemhauser et al., editor, Optimization. Elsevier, Amsterdam, 1989. 


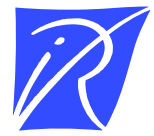

Unité de recherche INRIA Rocquencourt Domaine de Voluceau - Rocquencourt - BP 105 - 78153 Le Chesnay Cedex (France)

Unité de recherche INRIA Lorraine : LORIA, Technopôle de Nancy-Brabois - Campus scientifique 615, rue du Jardin Botanique - BP 101 - 54602 Villers-lès-Nancy Cedex (France)

Unité de recherche INRIA Rennes : IRISA, Campus universitaire de Beaulieu - 35042 Rennes Cedex (France)

Unité de recherche INRIA Rhône-Alpes : 655, avenue de l'Europe - 38330 Montbonnot-St-Martin (France)

Unité de recherche INRIA Sophia Antipolis : 2004, route des Lucioles - BP 93 - 06902 Sophia Antipolis Cedex (France)

INRIA - Domaine de Voluceau - Rocquencourt, BP 105 - 78153 Le Chesnay Cedex (France)

http://www.inria.fr

ISSN 0249-6399 